\title{
LA RESOLUCIÓN DE PROBLEMAS: UNA REVISIÓN ESTRUCTURADA
}

\author{
PERALES PALACIOS, F.J. \\ Departamento de Didáctica de las Ciencias Experimentales. Facultad de Ciencias \\ de la Educación. Campus Universitario de Cartuja. Granada.
}

\section{SUMMARY}

The aim of this paper is to review and organize the background, historic development and present situation of problemsolving research on Sciences and Mathematics.

\section{INTRODUCCIÓN}

En los últimos tiempos, los procesos cognitivos que responden al enunciado general de «resolución de problemas», y que han constituido tradicionalmente un apartado común a los tratados de psicología, han despertado un interés creciente entre los didactas de la física, ciencia que se presta excepcionalmente bien a esta clase de investigación educativa, dado el alto nivel de desarrollo teórico y práctico que la sustenta. De hecho, el papel jugado por la resolución de problemas en la enseñanza de la física está trasladándose desde constituir meros ejercicios de aplicación o cálculo complejo, hasta irse convirtiendo en un objetivo prioritario de la instrucción. El problema adquiere así una dimensión de actividad de enseñanza-aprendizaje, tanto de conceptos como de habilidades, y evaluadora no sólo de dicho aprendizaje sino de los propios mecanismos cognitivos puestos en juego por el educando.

\section{DEFINICIÓN Y CLASIFICACIÓN DE LOS PROBLEMAS}

Bajo el encabezamiento de resolución de problemas se reúnen tareas extremadamente diversas, Io que ha causado en gran medida la dificultad de su interpretación teórica (Cohen 1977). No obstante, creemos necesario distinguir en primer lugar lo que se entiende comúnmente por problema y por su resolución.

El «problema» podría ser definido genéricamente como cualquier situación prevista o espontánea que produce, por un lado, un cierto grado de incertidumbre y, por el otro, una conducta tendente a la búsqueda de su solución. En la vida ordinaria se resuelve un problema para obtener un resultado; por el contrario, en el contexto escolar el resultado importa poco (a menudo es conocido) y sí lo hace la propia resolución (Dumas-Carré 1987).

La «resolución de problemas» se utilizaría para referirse al proceso mediante el cual la situación incierta es clarificada e implica, en mayor o menor medida, Ia aplicación de conocimientos y procedimientos por parte del solucionador (Gagné 1965, Ashmore et al. 1979) así como la reorganización de la información almacenada en la estructura cognitiva (Novak 1977), es decir, un aprendizaje. La palabra «resolución» sirve para designar la actividad que consiste en resolver el problema desde la Iectura del enunciado, pudiendo establecerse una distinción entre el tratamiento logico-matemático y la propia actividad de resolución, analizada a menudo en términos de encadenamiento de procesos, y la solución o respuesta, producto de dicha actividad (Dumas-Caré 1987).

En cuanto a la clasificación de los problemas, puede hacerse atendiendo a diversos criterios:

a) Campo de conocimiento implicado (p.e., ciencia-no ciencia).

b) Tipo de tarea (cualitativa-cuantitativa).

c) Naturaleza del enunciado y características del proceso de resolución (problemas cerrados-abiertos).

Detengámonos en cada uno de estos apartados: 
a) Campo de conocimiento implicado.Como hemos señalado anteriormente, la principal diferencia entre los problemas que se plantean en la enseñanza de la ciencia y aquéllos que tienen lugar en la vida cotidiana estriba en que en el primer caso lo importante no es la obtención de la solución (a menudo conocida) sino más bien el proceso para llegar a ella; en cambio, ocurre lo contrario en los problemas cotidianos (p.e., reparación de un automóvil, confección de la declaración de la renta, etc.).

En otro orden de cosas, cabría diferenciar dentro del campo científico entre problemas de física, química, etc. como problemas semánticamente ricos por contraposición a otros tipos de problemas utilizados en psicología que obvian el contenido y se centran en las estrategias de resolución.

b) Tipo de tarea. Dentro ya del contexto de la enseñanza de las ciencias, se entiende por problemas cualitativos aquéllos que en su resolución no se precisa recurrir a determinaciones numéricas, debiendo resolverse đe forma verbal/escrita; normalmente se refieren a la interpretación científica de fenómenos reales y se les denomina con cierta frecuencia «cuestiones». Por el contrario, los problemas cuantitativos, o simplemente "problemas", exigen cálculos numéricos efectuados a partir de las ecuaciones correspondientes y de los datos disponibles en el enunciado.

c) En cuanto a la naturaleza del enunciado y características del proceso de resolución de problemas, los problemas cerrados son aquellas tareas que contienen toda la información precisa y son resolubles mediante el empleo de un cierto algoritmo por parte del solucionador. Los problemas abiertos, por el contrario, implican la existencia de una o varias etapas en su resolución que deben ser aportadas por el solucionador mediante una acción de pensamiento productivo (López 1989). Bajo este criterio, los problemas cualitativos pueden ser considerados en la mayoría de los casos como problemas abiertos y Ios cuantitativos como cerrados.

\section{VARIABLES A CONSIDERAR EN LA RESO- LUCIÓN DE PROBLEMAS}

Estas variables pueden agruparse en torno a:

- La naturaleza del problema.

- El contexto de la resolución del problema.

- El solucionador del problema (Cohen 1977).

a) La naturaleza del problema. Las variables que se contemplan fundamentalmente se refieren a los aspectos formales del problema tales como la precisión o univocidad, estructura, lenguaje, etc. del enunciado; complejidad y tipo de tarea requerida en la resolución; solución abierta o cerrada, conocida o desconocida; etc.

Para Dumas-Carré (1987), el enunciado de un problema comporta seis elementos:
* La descripción de un dispositivo y su funcionamiento eventualmente acompañado de un esquema.

* Las condiciones experimentales a que está sometido.

* Unas simplificaciones o modificaciones parciales que inscriben el fenómeno estudiado en un marco teórico simple.

* Unos datos, valores tomados por ciertas magnitudes físicas que se llaman a menudo «condiciones iniciales o condiciones en los límites".

* Una(s) pregunta(s).

* Unas consignas de repuesta más o menos implícitas interviniendo en la formulación de la pregunta.

b) El contexto de la resolución del problema. En este caso habría que reparar en aquellas variables intervinientes en el proceso de resolución sin tener en cuenta al propio solucionador. Así cabría hablar de la manipulación o no de objetos reales, la consulta o no de fuentes de información, la verbalización o no de la resolución, si se suministra o no el algoritmo puesto en juego, tiempo de resolución, etc.

c) El solucionador del problema. Finaimente incluimos aquí las características del solucionador tales como conocimiento teórico, habilidades cognitivas, creatividad, actitud, ansiedad, edad, sexo, etc. Igualmente se podría hablar de solucionador individual o grupal (Klausmeier y Goodwin 1975).

Cuando acometamos en el penúltimo apartado la perspectiva didáctica de la resolución de problemas, tendremos ocasión de ir analizando las aportaciones empíricas de diversos autores sobre resolución de problemas en ciencias.

\section{PERSPECTIVA HISTÓRICO-PSICOLÓGICA}

Bajo este epígrafe vamos a revisar someramente la historia reciente de las investigaciones sobre resolución de problemas, las cuales han tenido lugar fundamentalmente al amparo de la psicología experimental. Sólo en los últimos años ha tomado cuerpo una investigación específica de resolución de problemas en el marco de las ciencias experimentales o las matemáticas.

El abordaje de esta perspectiva de la resolución de problemas va a respetar el orden cronológico del surgimiento de las distintas corrientes psicológicas que la han acogido, es decir:

- Psicología conductista.

- Psicología de la Gestalt.

- Psicología cognitiva: teoría del procesamiento de la información.

- Psicología cognitiva: teoría de Piaget.

- Psicología cognitiva: constructivismo. 


\section{a) Psicología conductista}

De acuerdo con ésta, to que importa en el proceso de resolucion de problemas es la respuesta y su mecanismo de selección asociados con el estímulo presente en el problema. Las primeras investigaciones se basaron en la identificación -a través de la observación- de las estrategias de resolución de problemas empleadas por distintas personas en un intento de buscar similitudes entre ellas. Así Wallas (1926) describió las cuatro etapas siguientes: preparación (acumulación de información), incubación (marginación transitoria del problema), iluminación (un «darse cuenta» repentino) y verificación (hallazgo de la solución) (Mayer 1981).

En esta misma línea pueden situarse los trabajos de Polya $(1945,1968)$ sobre resolución de problemas matemáticos. La principal novedad es apuntar que una estrategia adecuada para resolver problemas considerados muy difíciles consiste en su fraccionamiento en subproblemas más simples que sí admiten una solución.

El motivoúltimo de este enfoque radica en la consiguiente necesidad de enseñar las estrategias de resolución de problemas presentes en las observaciones previas, pero en la mayoría de las ocasiones se obtuvieron resultados infructuosos.

\section{b) Psicología de la Gestalt}

Coincidiendo con las observaciones de Polya, aunque precursores en el tiempo, los psicólogos de la Gestalt detectaron la tendencia de los solucionadores a fraccionar los problemas en diversas etapas para intentar resolverlas posteriormente (p.e. Duncker 1945). La mayor contribución del enfoque gestáltico ha sido el énfasis puesto en la vertiente perceptual del proceso; para los seguidores de esta corriente la aprehensión apropiada de las partes del problema asegura que las «fuerzas de la organización" produzcan la solución. De cualquier modo, no se especifica con exactitud qué son esas fuerzas de organización. En cuanto al fracaso en la resolución de problemas se debe frecuentemente a la persistencia de un set rígido e inapropiado que puede estar causado por la experiencia previa o a la forma en que se expresan las instrucciones del problema. Ese set podría desvanecerse mediante las orientaciones del experimentador que permitan «recentrar» o dirigir la atención hacia los aspectos más significativos del problema.

Otra contribución novedosa de los téricos de la Gestalt como Duncker (1945) es la «valoración» de las posibles soluciones de un problema. Esto tiene una importancia especial en la vida cotidiana, donde diversas soluciones generan ventajas e inconvenientes, así como en la «toma de decisiones" consiguiente (Cohen 1977).

\section{c) Psicología cognitiva: teoría del procesamiento de la información}

Las teorías del procesamiento de la información describen la resolución de problemas como una interacción entre el «sistema de procesamiento de la información» del sujeto y un «ambiente de la tarea» tal como la des- cribe el experimentador. Este enfrentamiento produce en el solucionador una representación mental del problema denominada «espacio del problema» (Simon 1978) y que contiene el estado actual del problema, el estado final (o meta) y todos los estados intermedios. La resoIución de un problema conlleva una búsqueda -dirigiđa por el objetivo- a través del espacio del problema.

La incursión de la psicología cognitiva en el análisis de la resolución de problemas viene de la mano de la creación de los primeros ordenadores electrónicos (finales de la década de los cuarenta y comienzos de los cincuenta). Una de las principales utilidades de aquéllos era la de resolver problemas de complejidad creciente. Para ello se necesitaba dotar a los ordenadores de los siguientes recursos: un conjunto de almacenes de memoria y procesos de transformación, un conjunto de procedimientos para acceder a objetivos, un conocimiento verbal y un conjunto de estrategias generales, 0 heurísticas, que controlaran el proceso de resolución de problemas (Mayer 1981).

La investigación en este campo tecnológico -inteligencia artificial- tuvo su correspondiente reflejo en los estudios sobre resolución de problemas en humanos. Así nació eI «Solucionador General de Problemas» (SPG) de Ernst y NewelI (1969). Éstos crearon su modelo general de estrategia para la resolución de problemas sin tener en cuenta el contenido al que se aplicaban. Para su creación, tanto Emst y Newell como más tarde Newell y Simon (1972) se apoyaron en la verbalización de la resolución de problemas por parte de diversos solucionadores para extraer, seguidamente, la estrategia subyacente y tratar de generalizarla. Para Stewart y Atkin (1982), la importancia de este trabajo se ha dejado sentir en varias áreas generales:

* La elaboración de los conceptos del ámbito de la tarea (definición objetiva de problema) y el espacio del problema (representación interna del solucionador sobre el problema).

* El uso de un formato de «pensamiento» en voz alta como un método para indagar las estrategias utilizadas por el solucionador de un problema.

* El uso del modelo de computador y sistemas de producción para la representación de los pasos realizados en la resolución.

* El reconocimiento de que mientras puede haber muchas estrategias o heurísticas generales para la resolución de problemas, tales como el análisis de medios-fines, existe bastante evidencia que sugiere que las estrategias son específicas del contenido.

El modelo deI SPG tuvo su continuidad en las investigaciones psicológicas. Así, Atwood y Polson (1976) rediseñaron el SGP para resolver problemas relativos a recipientes de agua.

\section{d) Psicología cognitiva: teoría de Piaget}

Haciéndonos eco de la teoría dependiente de las etapas, según la formulación clásica de la misma, el individuo 
que accede a las operaciones formales sería capaz de resolver cualquier tipo de problema (Inhelder y Piaget 1955), independientemente de su contenido. No obstante, años más tarde Piaget (1970, citado en Pozo 1987) hubo de reconocer la influencia del contenido en la resolución de problemas formales. La perspectiva piagetiana o postpiagetiana pone su acento en la necesidad de potenciar el desarrollo cognitivo a través de la resolución de problemas (Pomes 1991).

Esta visión sobre la resolución de problemas ha sido revisada por los neopiagetianos como Pascual-Leone en términos de la necesaria adición de la «demanda-M» para la resolución de una tarea (cantidad de procesamiento de la información requerida por la tarea) (Niaz 1988).

\section{e) Psicología cognitiva: constructivismo}

El punto de partida de la toma de posición del constructivismo en el seno de la resolución de problemas hay que buscarlo en la dependencia entre dicho proceso y el contenido en el que se contextualiza el problema. Se confirmaba asi que el razonamiento no sólo tiene forma sino también contenido (Pozo 1987). Lo novedoso de este enfoque estribaba en el estudio de modelos de pensamiento circunscritos a las situaciones específicas de los problemas. Así ha llegado a considerarse la resolución de problemas independientemente de su estructura lógica y fuertemente dependiente de su representación mental y comprensión por parte del sujeto $\mathrm{y}$, en definitiva, de sus ideas previas sobre los conceptos implicados.

En cierta forma esta tendencia ha convergido con la deducida de la psicología del procesamiento de la información, en cuanto que ésta ha abordado el diseño de sistemas expertos que tratan de solucionar problemas específicos. Asimismo se ha acometido la comparación entre la resolución de problemas por parte de expertos y novatos, es decir, entre sujetos que difieren en la cantidad y calidad de sus preconcepciones (Simon y Simon 1978, Camacho y Good 1990). Como afirmara Novak (1977), una buena capacidad de resolución de problemas requiere conceptos bien diferenciados que sean relevantes para los problemas que se desea resolver.

Pozo (1987) señala una serie de presupuestos comunes a este tipo de estudios:

* La diferencia expertolnovato es básicamente una diferencia de conocimientos y no de procesos básicoso capacidades cognitivas.

* Esta diferencia de conocimientos es tanto cualitativa como cuantitativa; esto es, los expertos no sólo saben más que los novatos, sino que sobre todo tienen organi. zados sus conocimientos de una forma distinta.

* La pericia es un efecto de la práctica acumulada, esto es, un efecto del aprendizaje, desdeñándose, por tanto, los factores innatos y las posibles diferencias individuales.

* La pericia está circunscrita a áreas específicas de conocimiento, de forma que se es experto o no con respecto a algo. Un mismo sujeto puede tener grados diversos de pericia para problemas conexos de una misma área.

En cualquier caso y, en palabras del mismo autor, la metodología y resultados de este tipo de trabajos presentá algunas incertidumbres no clarificadas:

* ¿Cómo diferenciar entre expertos y novatos? No existe ningún patrón para clasificar de modo absoluto a los individuos en estos dos grupos.

* Ningún trabajo ha demostrado que expertos y novatos no difieran en sus procesos básicos de razonamiento.

* Dentro de un mismo nivel de pericia aparecen notables diferencias individuales que no reciben explicación.

Estas diferencias podrían dar lugar a dos tipos de interpretaciones con consecuencias distintas:

1) Los sujetos que difieren son igualmente expertos. En este caso, las diferencias halladas responden a la existencia de diversos modelos o sistemas expertos para la solución de un mismo problema. Esto estaría en línea con las posiciones piagetianas y, en concreto, con la propia epistemología del conocimiento científico.

2) Los sujetos que difieren, en realidad, no son igualmiente expertos, entendiendo la dimensión expertolnovato como un continuo. El problema radica en este caso en averiguar la causa de esta diferencia de pericia que podría ser debida a la vía instructiva o a la propia vía individual (entendida nuevamente en el sentido piagetiano).

Finalmente debemos mencionar la propuesta de Garret y otros (1990) para el análisis de las preconcepciones de los alumnos a través de la resolución de problemas.

\section{PERSPECTIVA EMPÍRICA: LA INVES- TIGACION EN RESOLUCIÓN DE PRO- BLEMAS}

En este cuarto apartado vamos a detenemos en la revisión de algunas investigaciones en torno a este tópico referido a ciencias y en algunos casos también a matemáticas. Ello lo haremos desde una triple vertiente:

- Variables en la resolución de problemas.

- Estrategias en la resolución de problemas.

- Didáctica, es decir, enseñanza de resolución de problemas, la resolución de problemas como instrumento de diagnóstico de las ideas previas y como instrumento de evaluación del aprendizaje.

a) Variables en la resolución de problemas

Los estudios empíricos en esta parcela suelen quedar dispersos dado el número de variables que pueden ser 
consideradas en un proceso tal, por lo que sería necesario emprender investigaciones del tipo «meta-análisis» (FitzGibbon 1984) para esclarecer, al menos en parte, el papel de las mismas.

De cara al solucionador de problemas, parece evidente la importancia del conocimiento especifico en la materia en que se inscriba el problema (Johnstone y Kellet 1980) o de la familiaridad con la tarea y de las habilidades cognitivas de aquél, ya sean entendidas éstas como inteligencia general (Klausmeier y Goodwin 1975) o en el sentido piagetiano del término (Champagne y Klopfer 1982, Lawson 1983), independientemente del énfasis que se le desee dar a cada versión en función del modelo didáctico asumido y de la riqueza semántica de los problemas propuestos (Kempa 1986, Saunders y Nathadas 1988). De una forma más explícita, Ferguson-Hessler y De Jong (1984) hablan de cuatro componentes del conocimiento: 1) conocimiento declarado (definiciones, teoremas, ecuaciones, etc.); 2) conocimiento de procedimiento (cómo aplicar una ecuación dada, cómo elegir una superficie gaussiana, etc.); 3) conocimiento de selección (cómo ser capaz de reconocer características relevantes de Ia situación dada y combinar éstas con las condiciones de validez de las fórmulas y procedimientos conocidos a fin de seleccionar uno o más para la resolución); 4) conocimiento de estrategia (sucesión de acciones que se realizan a fin de alcanzar una solución).

En cuanto a las dificultades que presentan los alumnos para resolver problemas de ciencias, Genyea (1983) ha señalado, junto a la ausencia de habilidades matemáticas específicas, una combinación de:

1) Deficiencias en la capaciđad de razonamiento formal.

2) Incapacidad o actitud negativa para construir una representación física apropiada dado un problema en forma escrita.

3) La creencia errónea de que para cualquier problema existe una fórmula o procedimiento donde introducir números.

4) La ansiedad sobre los problemas cuantitativos debido a dificultades pasadas con problemas de este tipo.

Otras dos dimensiones cognitivas no encasillables estrictamente en los ámbitos anteriores, esto es, el estilo cognitivo y la creatividad, han evidenciado igualmente una influencia sobre la capacidad de resolver problemas:

* El primero ha sido identificado como «dependenciaindependencia de campo» y medido a través del «test de figuras enmascaradas» (Witkin et al. 1971). Esta variable puede ser interpretada como la forma en que un individuo percibe la realidad, bien en los aspectos irrelevantes-los dependientes de campo-oen los más relevantes -los independientes-y ha sido relacionada con diversas variables educativas, tales como el nivel de desarrollo cognitivo o la resolución de problemas (López 1989, Garret 1989). En general, la independencia de campo viene a favorecer la resolución de problemas, aunque esta diferenciación depende de la naturaleza y contexto del problema (Corral 1982).
* En cuanto al papel de la creatividad, existen diversos estudios dedicados a establecer tal relación. En primer lugar debería especificarse lo que se entiende por creatividad o «pensamiento divergente», como se le ha venido a denominar también en la literatura especializada. Como denominador común aparecen la fluidez, la flexibilidad, la originalidad y la elaboración (Guilford y Hoepfner 1966). Garret $(1987,1988)$ ha añadido el factor de utilidad, considerando la creatividad como una función de ésta y de la originalidad.

La creatividad aparece como un buen predictor de la resolución de problemas en el caso de que sean de naturaleza abierta, pero no tiene por qué serlo cuando se trate de problemas de solución lógica ya conocida.

* En cuanto al sexo, parece ser que la diferencia entre varones y hembras en su capacidad de resolver problemas varía desde la infancia a la adolescencia, primando a las segundas en los intervalos de menor edad y a los primeros a partir de la adolescencia (Bethencourt y Torres 1987).

En la revisión del papel de la naturaleza del problema, se ha descrito la influencia del conocimiento del resultado previamente a su resolución (Perales y Cervantes 1984) o a posteriori (Escudero 1979). Con respecto al enunciado, se han elaborado instrumentos para su evaluación (Fernández et al.1984), se ha investigado el nivel de información suministrado (Kempa 1986) o la propia lectura del mismo (Fauconnet 1984).

\section{b) Estrategias de resolución de problemas}

En este segundo apartado hemos recopilado estudios que ponen de manifiesto las estrategias desarrolladas por los resolvedores de una forma espontánea -es decir, no producidas por la instruccion-y que han sido denominados «naturalísticos» o «descriptivos» (Reif 1983). Para ello, dichos estudios recurren frecuentemente a técnicas de verbalización (thinking aloud) (Kramers-Pals et al. 1982, Landa 1976) y, en menor medida, a un estudio cualitativo de la resolución de problemas escrita. Aunque la primera técnica resulta más representativa del pensamiento del individuo, lleva implícitas algunas limitaciones como son que la propia verbalización no refleja fielmente el pensamiento, la no conciencia de algunos pasos dados por el solucionador y la descoordinación entre la velocidad de pensamiento y de verbalización (Kramers-Pals et al. 1982, Dumas-Carré 1987).

La descripción de Ias estrategias utilizadas ha de ser adscrita a unas fases comunes a la resolución de problemas previamente aceptadas y que normalmente se identifican con un sistema heurístico.

* Stewart y Atkin (1982) se refieren a los intentos de diversos autores por encontrar estrategias generales de resolución de problemas entre los individuos, las cuales incluirian:

- Análisis de «medios-fines».

- Planteamiento.

- Utilización de metas y submetas. 
* Kramers-Pals et al. (1982) revisaron las dificultades de los estudiantes en torno a:

- Análisis del problema.

- Planificación del proceso de resolución de problemas.

- Ejecución de operaciones rutinarias.

- Chequeo de la respuesta e interpretación de los resultados.

* Alonso et al. (1988) identificaron un total de doce conductas diferentes de niños de educación primaria al resolver problemas en el siguiente orden de frecuencia decreciente:

- Cálculos operatorios.

- Desconcierto.

- Lectura del problema.

- Escribir datos.

- Formulación de hipótesis.

- Resolución.

- Bloqueo o ausencia de conducta.

- Rectificación.

- Utilización de datos irrelevantes.

- Representación de datos.

- Anticipación de los resultados.

- Comprobación.

* Otra forma ya comentada de afrontar este tópico es la separación entre expertos o buenos resolvedores y novatos o malos resolvedores y comparar las estrategias de ambos grupos. Así, Kempa y Nicholls (1983) adoptaron este procedimiento de cara a la comparación entre las estructuras cognoscitivas de dos muestras de estudiantes. Larkin (1981) utilizó un programa de ordenador para simular el comportamiento en la resolución de problemas por parte de novatos y para comprender mejor la transición novato $\rightarrow$ experto. Esta misma línea de actuación aparece comúnmente entre las investigaciones sobre concepciones espontáneas donde, mediante la indagación en las estrategias de resolución de problemas de los novatos, intentan poner de manifiesto los obstáculos cognitivos -esto es, tales concepciones- para encontrar la solución correcta (Fauconnet 1984).

De acuerdo con la base de conocimiento precisa para una correcta resolución de problemas y, partiendo de la propuesta de Fergusson-Hessler y De Jong (1984) descrita anteriormente, sería posible extrapolar las siguientes conclusiones a partir del análisis de los datos sobre resolución de problemas de una muestra de alumnos:

EI conocimiento declarado está presente generalmente de forma reproducible, pero a menudo no es aplicable a un problema dado $\mathrm{y}$, a veces, contiene elementos incorrectos.

-El conocimiento de procedimiento es a menudo incompleto.

- El conocimiento de selección está implícito o ausente.

- La estrategia usada es la de «patada y embestida».

\section{c) Perspectiva didáctica}

De la revisión de los estudios que ponen su énfasis en la aplicación de la resolución de problemas al campo de la enseñanza, sobresalen dos tendencias. En primer lugar, la que se centra en la necesidad de resolver problemas de un modo eficiente, ya sea por considerarlo una importante meta didáctica o por buscar la comprensión conceptual y la competencia en las habilidades correspondientes. En segundo término surge el papel de la resolución de problemas como instrumento de diagnóstico de errores conceptuales y concepciones alternativas, así como para la evaluación del propio aprendizaje adquirido odel cambio conceptual. En cualquier caso, la realidad pone en evidencia la práctica ausencia de metodologías específicas para la resolución de problemas en los programas oficiales y en los libros de texto educativos (Dumas-Carré 1987).

Comenzaremos por revisar algunos trabajos dedicados a la enseñanza de resolución de problemas:

* Varios autores se han preocupado de recomendar o verificar estrategias para estimular la solución de problemas creativos (Klausmeier y Goodwin 1975).

* En cuanto a las estrategias para resolver problemas gı̀nerales, Landa (1976) estableció una distinción entre los diferentes métodos de instrucción para la resolución de problemas que se representa en la tabla I. Ya que los métodos algorítmicos garantizan una solución correcta a los problemas, Landa se pregunta por qué no se enseñan siempre tales métodos. La respuesta para este autor consiste en la imposibilidad de disponer de algoritmos para todos los problemas, ya que en muchos casos no se conoce de antemano cómo resolverlos, para lo cual hay que recurrir a reglas heurísticas. Una amplia panorámica del uso de algoritmos en la resolución de problemas puede encontrarse en el resumen de las comunicaciones presentadas al « 190th National Meeting of the American Chemical Society, Symposium on the Use of AIgorithm in Problem Solving", celebrado en Chicago el 9 de septiembre de 1985 (Journal of Chemical Education, 1987. Vol. 64(6), pp. 509-523; Apuntes de Educación, 1991 núm. 41).

\section{Tabla I}

Métodos de resolución de problemas versus solución a los mismos (Landa 1976, p. 394).

\begin{tabular}{lllll}
\hline & $\begin{array}{l}\text { Algo- } \\
\text { ftrmicos }\end{array}$ & $\begin{array}{l}\text { Semi- } \\
\text { algorítmicos }\end{array}$ & $\begin{array}{l}\text { Semi- } \\
\text { heurísticos }\end{array}$ & $\begin{array}{l}\text { Heurís- } \\
\text { ticos }\end{array}$ \\
$\begin{array}{l}\text { Todos los sujetos en- } \\
\text { cuentran una solución }\end{array}$ & Sí & Sí & Sí & No \\
$\begin{array}{l}\text { Todos encuentran una } \\
\text { solución correcta }\end{array}$ & Sí & Sí & No & No \\
$\begin{array}{l}\text { Todos los resuelven de } \\
\text { la misma manera }\end{array}$ & Sí & No & No & No \\
L.............. & & & &
\end{tabular}


En cualquier caso, son los métodos heurísticos los más recomendados en el ámbito investigador, presentados como modelos prescriptivos y con fases en gran medida comunes a las propuestas pioneramente por Polya (definir, planificar, ejecutar y retrotraer) e incidiendo en las bases conceptuales y procesuales precisas (estas últimas deducidas a partir de los estudios con expertos).

Los trabajos de investigación difieren entre los que se quedan en simples propuestas (Mettes et al. 1980, 1981, Reif 1981, 1984, Reif y Heller 1982, Champagne et al. 1982, Genyea 1983, Razafindrakoto 1984, FergussonHessler y Larcher 1984, Reif y Caillot 1984, Ryan 1985, Fasching y Erickson 1985, Cornelius et al. 1985) y aquéllos que evalúan tales propuestas (Larkin, citado en Stewart y Atkin 1982, Stewart 1982, vanWeeren et al. 1982, Cook 1982, Kramers-Pals et al, 1983, vanWeeren 1984, Faucher 1984, Drake 1985, Gascón 1985, Ott 1987, Dumas-Carré 1987, Ramírez 1990) con resultados mayoritariamente positivos para los métodos introducidos.

Pasando seguidamente al papel de Ios problemas como instrumento de evaluación, poco material bibliográfico puede hallarse al respecto. Existe un consenso entre los educadores de ciencias respecto del uso de los problemas como elemento más o menos significativo dentro de las pruebas de evaluación; no obstante, en una gran mayoría de las ocasiones se les pide a los alumnos que resuelvan problemas-tipo similares a los afrontados en el aula. Esta costumbre favorece la evaluación de los saberes declarativos (las definiciones o los enunciados de leyes - los procedimientos de resolución) en detrimento de los saberes procedimentales (Dumas-Carré 1987).

En cuanto a la utilización de la resolución de problemas dentro del objetivo de detectar preconcepciones o errores conceptuales - dentro del marco del modelo constructivista- tampoco el panorama es más alentador. Los problemas, sobre todo cualitativos y de bajo nivel de complejidad, son incluidos ocasionalmente como parte de los pretests habituales. En cambio, la resolución de problemas puede tener una incidencia más activa en el caso de las estrategias instructivas destinadas a conseguir el deseado cambio conceptual. Así, en un estudio de síntesis de propuestas instructivas (Perales 1988), hallamos las siguientes, donde sería factible reconocer o incluir la resolución de problemas:

- Animar a contrastar los preconceptos con las explicaciones científicas, probando los alumnos sus propios modelos en clase.

- Conseguir el cambio conceptual, presentando las insuficiencias de los preconceptos de los alumnos y las ventajas de la solución propuesta.
- Aplicación del nuevo modelo conceptual.

\section{PERSPECTIVA FUTURA}

Las páginas anteriores deben habernos servido para tomar conciencia de lo lejos que nos encontramos de un estado consensuado en torno a las variables que intervienen en la resolución de problemas y a las estrategias tendentes a su mejora. Esta situación es, a nuestro juicio, un síntoma más de la aparente transición por la que atraviesa la didáctica de las ciencias, con un modelo didáctico, el constructivista, sometido a ciertas tensiones en aras de abrirlo a otros parámetros educativos y de articularlo en la práctica instructiva, antes de poder hablar de una «teoría constructivista de la enseñanzaaprendizaje». Y en este movimiento, la resolución de problemas se halla en el ojo del huracán cuando determinadas voces reclaman desde la filosofía de la ciencia o desde su didáctica que el objetivo de la ciencia es «resolver problemas» o que la enseñanza debe concebirse como un proceso de investigación, respectivamente. En cualquier caso, lo que sí parece demostrado es el importante papel desempeñado por la resolución de problemas en cualquier enfoque asumido para la enseñanza de las ciencias y de las matemáticas, especialmente entre las más «duras» (física, química y matemáticas, aunque con prometedoras perspectivas en biología y geología). Este reconocimiento debería llevar aparejado una mayor atención entre los investigadores para dar respuesta a numerosas preguntas que aún quedan en el aire. Finalizaremos sugiriendo algunos interrogantes:

* ¿Qué variables son más relevantes para una resolución de problemas efíciente, las dependientes o las independientes del contexto?

* ¿Qué tipos de estrategias realistas habría que usar para resolver problemas de diferente naturaleza?

* ¿Cómo integrar la resolución de problemas en una perspectiva constructivista de la enseñanza-aprendizaje?

* ¿Cabe establecer una diferenciación drástica, desde el punto de vista didáctico, entre los trabajos prácticos y la resolución de problemas?

* ¿Qué lugar debe ocupar la resolución de problemas en la evaluación del aprendizaje?

* ¿Cómo incorporar los resultados de la investigación educativa en los manuales de resolución de problemas? 


\section{REFERENCIAS BIBLIOGRÁFICAS}

ALONSO, V.,GONZÁLEZ, A. y SAENZ, O., 1988. Estrategias operativas en la resolución de problemas matemáticos en el ciclo medio de la EGB, Enseñanza de las Ciencias, Vol. 6 (3), pp. 251-269.

ASHMORE, A.D.,FRAZER, M.J. y CASEY,R.J., 1979. Problem solving and problem solving networks in chemistry, J. Chem. Educ, Vol. 56, pp. 377-379.

ATWOOD, M.E. y POLSON, P.G., 1976. A process model for water jug problems, Cognitive Psychology, Vol. 8, pp. 191216.

BETHENCOURT, J.T. y TORRES, E., 1987. La diferencia de sexo en la resolución de problemas aritméticos: un estudio transversal, Infancia y Aprendizaje, Vol. 38, pp. 9-20.

CAMACHO, M. y GOOD, R., 1990. Problem solving and chemical equilibrium: Successful versus unseccessful performance, J. Res. Sci. Teach, Vol. 26 (3), pp. 251-272.

COHEN, G., 1977.The psychology of cognition. (Academic Press: Londres). Trad. española: 1983, Psicología cognitiva. (Alhambra: Madrid).

COOK, D.M., 1982. Videotaped problem solutions for introductory physics, Am. J. Phys, Vol. 50 (3), pp. 268-269.

CORNELIUS, R., CABROL, D. y CACHET, C., 1985. Student use of computers for solving problems: Tools or crutches?, J. Chem. Educ, Vol. 62 (12), pp. 1094-1095.

CORRAL, A., 1982. La influencia del estilo cognitivo «dependencia-indepedencia de campo» en la resolución de dos problemas de física, Infancia y Aprendizaje, Vol. 18, pp. $107-123$

CHAMPAGNE, A.B., KLOPFER, L.E. y GUNSTONE, R.F., 1982. Cognitive research and the design of science instruction. Educational Psychologist, Vol, 17 (1), pp. 31-53.

CHAMPAGNE, A.B. y KLOPFER, L.E., 1982. A causal model of students achievement in a college physics course, J. Res. Sci. Teach, Vol. 19, pp. 299-309.

DRAKE, R.F., 1985. Working backwards is a forward step in the solution of problems by dimensional analysis, $J$. Chem. Educ, Vol. 62, p. 414

DUMAS-CARRE, A., 1987. La resolution de problemes en Physique au Licée. Tesis doctoral. Universidad de París 7.

DUNCKER, R., 1945. On problem solving, Psychological Monographs, Vol. 58 (5), p. 270.

ERNST, G.W. y NEWELL, A., 1969. GPS: A case study in generality and problem solving. (Academic Press: Nueva York).

ESCUDERO, T., 1979. La evaluación periódica como estímulo didáctico. (Servicio de Publicaciones del MEC.: Madrid).

FASCHING, J.L. y ERICKSON, B.L., 1985. Group discussions in the chemistry classroom and the problem-solving skills of students, J. Chem. Educ.,Vol. 62 (10), pp. 842-846.

FAUCONNET, S., 1984. Étude de resolution de problèmes analogues. Atelier International d' été: Recherche en didactique de la Physique. La Londe les Maures (1983). (CNRS.: París), pp. 261-269.

FAUCHER, G., 1984. Resolution des problèmes et enseignement individualisé, Atelier International d'été: Recherche en didactique de la Physique. La Londe les Maures (1983). (CNRS: París), pp. 297-302.
FERGUSON-HESSLER, M. y DE JONG, T., 1984. On success and failure in the solving of problems in electricity and magnetism, International Summer Workshop: Research on Physics Education. La Londe les Maures (1983). (CNRS.: París), pp. 271-279.

FERGUSON-HESSLER, M. y LARCHER, C. (leaders), 1984. Problem-solving (workshop 9), International Summer Workshop: Research on Physics Education. La Londe les Maures (1983). (CNRS: Paŕs), pp. 601-604.

FERNÁNDEZ, E., PERALES, F.J. y PLAZA, R., 1984, Una experiencia sobre didáctica de los problemas, Cuadernos de Física y Química, Vol. 5, pp. 47-50.

FITZ-GIBBON, C.T., 1984. Meta-analysis: an explanation, Brit. Educ. Res. J., Vol. 10 (2), pp. 135-144.

GAGNÉ, R.M., 1965. The conditions of learning. (Holt, Rinehart and Winston: Nueva York). Trad. española: 1971, Las condiciones del aprendizaje. (Aguilar: Madrid).

GARRET, R.M., 1987. Issues in science education: problemsolving, creativity and originality, Int. J. Sci. Educ, Vol. 9 (2), pp. 125-138.

GARRET, R.M., 1988. Resolución de problemas y creatividad implicaciones para el currículo de ciencias, Enseñanza de las Ciencias, Vol. 6 (3), pp. 224-230.

GARRET, R.M., 1989. Problem-solving and cognitive style, Res. Sci. Technol. Educ., Vol. 7 (1), pp. 27-44.

GARRET, R.M., SATERRLY, D., GIL, D. y MARTÍNEZ, I. 1990. Turning exercices into problems: An experimental study with teachers in training, Int.J.SCi.Educ., Vol. 12 (1), pp. 1-12.

GASCÓN, J., 1985. El aprendizaje de la resolución de problemas de planteo algebraico, Enseñanza de las Ciencias, Vol. 3 (1), pp. 18-27.

GENYEA, J., 1983. Improving student's problem-solving skills. A methodological approach for a preparatory chemistry course, J. Chem. Educ, Vol. 60, pp. 478-481.

GIL, D., MARTÍNEZ, I. y SENENT, F., 1985. La resolución de problemas de física como investigación: resultados experimentales obtenidos con alumnos de enseñanza media. Comunicación al/Congreso Internacional sobre lnyestigación en Didáctica de las Ciencias y de las Matemáticas. Barcelona.

GIL, D., MARTÍNEZ, J. y SENENT, F., 1988. El fracaso en Ia resolución de problemas de f́́sica: una investigación orientada por nuevos sipuestos, Enseñanza de las Ciencias, Vol. 6 (2), pp.131-146.

GUILFORD, J.P. y HOEPFNER, R., 1966. Structure of-intellect factors and their tests. Report of the psychological laboratory. 36. (University of Southern Califomia Press: Los Angeles).

INHELDER, B. y PIAGET, J., 1955. De la logique de l'enfant a la logique del' adolescent. (Presses Universitaires de France: París). Trad. española: 1985, De la lógica del niño a la lógica del adolescente. (Paidós: Barcelona).

JOHNSTONE, A.H. y KELLET, N.C., 1980. Leaming difficulties in school science: Towards a working hypothesis, Eur.J.Sci. Educ, Vol. 2 (2), pp. 175-181.

KEMPA, R.F. y NICHOLS, C.E., 1983. Problem solving ability and cognitive structure. An exploratory investigation, Eur. J. Sci. Educ, Vol. 5 (2), pp. I71-184. 
KEMPA, R.F., 1986. Resolución de problemas de química y estructura cognoscitiva, Enseñanza de las Ciencias, Vol. 4 (2), pp, 99-110.

KLAUSMEIER, H.J. y GOODWIN, W., 1975. Learning and human abilities educational psychology. (Harpeer and Row, Publishers: Nueva York, 4a. ed.). Trad. española: 1977, Psicologia educativa: habilidades humamas u aprendizaje. (Harla: México).

KRAMERS-PALS, H., LAMBRECHTS, J. y WOLFF, P.J., 1982. Recurrent difficulties: Solving quantitative problems, J. Chem. Educ., Vol. 59, pp. 509-513.

LANDA, L.N., 1976. The ability to think-How can it be taught? Soviet Education, Vol. 18 (5), pp. 4-66. Trad. española: A. Pérez y J. Almaraz (ed.), 1982, Lecturas de aprendizaje y enseñanza. (Zero: Madrid).

LARKIN, J.H., 1981. Cognition of leaming physics, Am.J.Phys, Vol. 49 (6), pp. 534-541.

LAWSON, A.E., 1983. Predicting science achievement: The role of developmental level, disembedding ability, mental capacity, prior knowledge, and beliefs, J. Res. Sci. Teach, Vol. 20 , pp. 117-129.

LÓPEZ, F., 1989. Dependencia-independencia de campo y educación científica, Revista de Educación, 289, pp. 235258.

MAYER, R.E., 1981. The promise of cognitive psychology. (W.H. Freeman and Company: EEUU). Trad. española: 1985, El futuro de la psicologia cognitiva. (Alianza Editorial: Madrid).

METTES,C.T.C.W. et al., 1980. Teaching and learning problem solving in science. Part I: A general strategy, $J$, Chem. Educ, Vol. 57 , pp. 882-885.

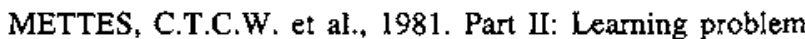
solving in a themodynamics course, J. Chem. Educ., Vol. 58, pp. 51-55.

NEWELL, A. y SIMON, H.A., 1972. Human problem solving. (Englewoods Cliffs, Prentice-Hall: Nueva Jersey).

NÍAZ,M., 1988. The information-processing demand of chemistry problems and its relation to Pascual-Leone's functional M-capacity, Int. J.Sci. Educ, Vol. 10 (2), pp. 23I-238.

NOVAK, J.D., 1977. A theory of education. (Cornell University Press: Ithaca). Trad. española: 1982, Teoría y práctica de la educación. (Alianza: Madrid).

OTT, M.B., 1987. Enseñanza por medio de solución de problemas, en: V.M. Candaut (ed.), La didáctica encuestión. Investigación y Enseñanza. (Narcea: Madrid).

PERALES, F.J. y CERVANTES, A., 1984. Influencia del conocimiento del resultado numérico en la resolución de problemas, Enseñanza de las Ciencias, Vol. 3, pp. 97-101.

PERALES, F.J., 1988. La instrucción científica en el marco de un modelo constructivista para la enseñanza, Revista de Educación de la Universidad de Granada, Vol. 2, pp. 115 133.

PIAGET, J., 1970. L'evolution intellectuelle entre l'âdolescence et l'âge adulte. Raport sur le llI Congrés International FONEME sur la fromation humaine a l'áge adulte. (Milán)

POL YA, G., 1945. How to solve it. (Princeton University Press: Nueva Jersey). Trad. española: 1965, Cómoplantear y resolver problemas. (Trillas: México).

POLYA, G., 1968. Mathematical discovery. (Wiley: Nueva York).

POMES, J., 1991. La meiodología de resolución de problemas y el desarrollo cognitivo: un punto de vista postpiagetiano, Enseñanza de las Ciencias, Vol. 9 (1), pp. 78-82.
POZO, J.I., 1987. Aprendizaje de la ciencia y pensamiento causal. (Visor: Madrid).

RAMÍREZ, J.L., 1990. La resolución de problemas de física y química como investigación en la enseñanza media: un instrumento de cambio metodológico. Tesis doctoral. (Universidad Autónoma de Barcelona).

RAZAFINDRAKOTO, E., 1984. La resolution des problèmes de physique dans son lieu avec la formation des maîtres. Atelier International d'été: Recherche en didactique de la Physique. La Londe les Maures (1983). (CNRS: París), pp. 517.522 .

REIF, F., 1981. Teaching problem-solving. A scientific approach, The Physics Teacher, Vol. 19, mayo, pp. 310-316.

REIF, F. y HELLER, J.I., 1982. Knowledge structure and problem solving in physics, Educational Psychologist, Vol. 17, pp. 102-127.

REIF, F., 1983. How can chemists teach problem solving? Suggestions derived from studies of cognitive processes, $J$. Chem. Educ, Vol. 60 (11), pp. 948-953.

REIF, F., 1984. Comprendre et enseigner la resolution de problèmes en physique. Atelier International d'été: Recherche en didactique de la Physique. La Londe les Maures (1983). (CNRS.: París), pp. 3-13.

REIF, F. y CAILLOT, M. (leaders), 1984. Problem solving (workshop 3). International Summer Workshop: Research on Physics Education. La Londe les Maures (1983). (CNRS.: París), pp. 544-552.

RYAN, C., 1985. Innovation in the curriculum: Introduction of the problem-solving in primary education. I Congreso Internacional sobre Investigación en Didáctica de las Ciencias y las Matemáticas. Barcelona.

SAUNDERS, W.L, y NATHADAS, J.J., 1988. The effect of task content upon proportional reasoning, J.Res. Sci.Teach, Vol. 25 (1), pp. 59-68.

SIMON, H.A., 1978. Information-processing theory of human problem solving, en Estes W.K. (ed.), Handbook of Learning and Cognitive Processes. Vol. 5: Human Information Processing. (Hillsdale: Nueva Jersey). Trad. española: $L a$ teoría del proce samiento de la información sobre la solución de problemas, en: Carretero, M., y García,J.A., 1984, Lecturas de Psicologíay Pensamiento. (Alianza Psicología: Madrid), pp. 197-219.

SMON, H.A. y SIMOND, 1978. Individual differences in solving physics problems en Siegier(ed.), Children's Thinking: What develops? (Lawrence Erlbaum. Hillsdale: Nueva Jersey).

STEWART, J., 1982. Two aspects of meaningful problem solving in science, Science Education, Vol. 66 (5), pp. 731749 .

STEWART, J. y ATKIN, J.A., 1982. Information processing psychology: A promising paradigm for research in science teaching, J. Res. Sci. Teach, Vol. 19 (4), pp. 321-332.

WALLAS, G., 1926. The art of thought. (Harcourt: Nueva York).

WEEREN, J.H.P, vAN et al., 1982. Teaching problem-solving in physics: A course in electromagnetism, Am.J.Phys, Vol. 50, pp. 725-732.

WEEREN, J.H.P. VAN, 1984. Route mapping and reflection in the process of leaming problem solving. International Summer Workshop: Research on Physics Education. La Londe les Maures (1983). (CNRS: París), pp. 257-260.

WITKIN, H.A. et al., 1971. A manual for the embedded figures tests. (Consulting Psychologists Press: Palo Alto). 